\title{
OBSERVATIONS OF SPACE DEBRIS ${ }^{1}$
}

\author{
L. G. TAFF ${ }^{2}$ \\ Lincoln Laboratory, M.I.T., Lexington, MA 02173-0073 U.S.A.
}

\begin{abstract}
Optical observations of near Earth and deep-space debris conducted at M.I.T.'s artificial satellite observatory will be discussed. A brief review of observing technique, regions of high debris density, and amount of debris in orbit will be given. The unique, duplex facilities of the observatory allow the discrimination of debris from meteors, the construction of an orbital element set, and real-time identification of cataloged artificial satellites. Near-Earth debris is present in large numbers in all the popular near-Earth orbits; at least 5-6 times the 5000-6000 objects in the NORAD catalog. In deep-space, the new presence of Ariane-related debris adds significantly to the existing population which is treble that cataloged by NORAD.
\end{abstract}

\section{INTRODUCTION}

Several United States government agencies are interested in deep-space satellite debris. Examples include the U.S. Air Force, whose responsibility it is to track all artificial satellites, and the National Aeronautics and Space Administration. NASA's principal concern is manned spacecraft protection. Some summaries of observational work already conducted are in Taff et $\underline{\text { al }}$. (1985), Taff and Jonuskis (1986), and Taff (1986). Additional documentation can be found in several Lincoln Laboratory Project Reports. Photometric calibrations are dealt with in Yakutis, Taff, and Sayer (1986) and Taff and Jonuskis (1987). Astrometric calibration and celestial mechanics analysis is in Taff (1985). Search theory for artificial satellites is discussed in Taff $(1987 a, b)$ as well as in Taff (1979) and (1982). For applications to asteroids see Taff (1981) and Taff (1984).

Orie result of optical searches sponsored by the Air Force and NASA, as well as routine detections by the Space Surveillance Network (including its radars and missile warning radars), is that there are now a large number of objects in an "Ariane-type" orbit. By this I mean $\mathrm{n} \sim 2$ rev/day, e $\sim 0.7$, and $\mathrm{i} \sim 7^{\circ}$. There are at least $\mathbf{2 5 0}$ pieces now in this type of orbit. The bulk of these could not have been in orbit three years ago for searches conducted then did not find them in

1. This work sponsored by NASA under contract NAS-9-17138.

2. Now at Space Telescope Science Institute operated by AURA, Inc. for NASA 
statistically significant numbers. Presumably the source of these objects is the recent explosions of Ariane-related launch vehicles (one on July 2, 1985 and one on March 18, 1986). One section of this paper describes them in detail (see also Taff 1987c).

Another derivative of these searches are new, more firmly grounded estimates of the debris population. Near-Earth debris (to $1 \mathrm{~cm}$ ) must be present at 5-6 times the NORAD catalog numbers. Similarly, deep-space debris triples the NORAD entries.

\section{BACKGROUND}

Descriptions of the observatories used for most of the search effort have already been published. Hence, I shall be extremely brief concerning them herein. However, there are two systematic corrections of importance which need to be discussed. This is done in the next section.

The Experimental Test System (in New Mexico) of the Ground-based Electro-Optical Deep Space Surveillance (GEODSS) network was built by and is operated by the Space Surveillance Group of Lincoln Laboratory (MIT). The interested reader can consult Weber (1979a,b,) and Taff (1981), and the references already cited for details about the observatory. Its primary function is to search for and track artificial satellites in deep space orbit. Under dark skies, and using video integration techniques, a limiting magnitude of $B \simeq 17^{\mathrm{m}}$ can be reached. This is the $50 \%$ probability of detection point. A poorly reflecting (albedo $=0.1$ ) piece of debris at opposition, but this faint, would have an effective radius of $\simeq 0.2 \mathrm{~m}$ at the geosynchronous distance.

After a proof-of-concept test in the winter of 1979 (the only deep, extensive optical search ever performed; Taff 1979); the Air Force decided that a computer-controlled, electro-optically equipped observatory (as the Experimental Test System was) could not only respond in real-time to tracking requests but would also complement the radar component of the Space Surveillance System. Hence, the Air Force contracted to have "replicas" of the observatory made and distributed world-wide. These formed the GEODSS network and are part of its Spacetrack system.

Descriptions of these observatories may be found in Beatty (1982). Site locations include New Mexico, Hawaii, South Korea, Diego Garcia (all operational), and the eastern Atlantic (planned). In general, the GEODSS instrumentation is significantly less sensitive than its prototype (perhaps $B \sim$ 15.5). Its search rate is four times higher though ( 600 square degrees per hour vs $150)$.

Over the 1986/1987 winter all four GEODSS sites were used in an extensive, coordinated search effort. The Baker-Nunn sites at St. Margarets, Canada and San Vito, Italy were incorporated in to this effort too. A smaller scale GEODSS/Baker-Nunn search in the winter of 1985 serves as part of the baseline for this report as does a NASA-sponsored search conducted at the Experimental Test System in October, 1985. Data from other optical searches have also been used in forming the conclusions of this paper. Finally, a large number and variety of radars, such as the FPS-85 at Eglin Air Force Base (Florida) and the NAVSPASUR continental fence, have contributed to the data base utilized in this paper. 


\section{SYSTEMATIC CORRECTIONS}

Optical observations of near-Earth satellites must be performed during twilight. Only then will near-Earth artificial satellites be illuminated while the observatory is in darkness and the background sky (actually foreground atmospheric scattering) will be dim. Thus, the phase angle is always near $90 \mathrm{o}$ and the search direction is always near the zenith. Experimentation shows that dawn is much superior to dusk (1-2 ${ }^{\mathrm{m}}$ of decreased extinction). These constraints mean that there are two substantial corrections which we need to incorporate. The first allows for the decreased viewing time of objects very near the Earth. They are eclipsed by the Earth's shadow for most of the observing interval. Hence, assuming that the object's flight overhead is uncorrelated with twilight (as it must be over the course of years), the few we do see at the lower elevations are representative of a much larger population which is generally invisible to us (see Fig. 1). The mathematical form of this systematic adjustment factor is simple to compute. Let $\mathrm{H}_{\mathrm{S}}$ be the height of the Earth's shadow at the commencement of observing (at dawn; at dusk the situation is reversed). Also let $\mathrm{H}_{\mathrm{e}}$ be the shadow height at the termination of the observing. Then the probability of detection, $P$, owing solely to eclipse/no eclipse considerations for an artificial satellite at height $\mathrm{H}$ is

$$
P=\begin{array}{ll}
1 & \text { for } H>H_{s} \\
\left.\left(H-H_{e}\right) / H_{B}-H_{e}\right) & \text { for } H \in\left[H_{e}^{, H_{s}}\right] \\
0 & \text { for } H<H_{e}
\end{array}
$$

The weights we need to apply are $1 / P$ if $P \neq 0$ and 0 if $P=0$. The assumption has been made that the rate of change of $\mathrm{H}$ with time is constant.

The second correction has to do with detectability and its variations. As an example, as the observing proceeds the sky lightens because the Sun is rising (the reverse occurs at dusk). This reduces our chances of seeing any faint object but does not substantially reduce our probability of detecting a cataloged satellite (because they are, in general, larger). Another problem is that the videotapes are recorded during twilights of differing quality. Once again, if the meteorological conditions are unfavorable, the statistics are systematically biased causing discrimination against pieces of debris-not the cataloged objects. To be complete we should have a method that puts all the videotapes on the same footing and corrects for variations within an observing run.

The problem is akin to Trumpler's when he discovered interstellar extinction. Trumpler however, had an advantage--as a first approximation he could assume that all galactic clusters had the same linear diameter. In the present instance a knowledge of the debris luminosity function is required to disentangle the extinction effects. Of course we have no such information precisely because of our observational difficulty. It is my estimate that could this correction be incorporated, then the observed 3:1 ratio of counts (near-Earth debris: NORAD catalog) would be doubled. Observations by others at a variety of wavelengths supports the $6: 1$ ratio. 


\section{DEEP-SPACE DEBRIS}

The systematic correction for sensitivity losses must be considered when examining deep-space debris searches too. In this area of artificial satellite observing enough different telescopes exist to more accurately quantify it. Above $10-20 \mathrm{~cm}$ in size, the number of deep-space debris pieces is at least triple the number of satellites cataloged by NORAD. As is the case for near-Earth debris, deep-space is concentrated in the orbital classes most commonly utilized by deepspace payloads and transfer rockets. The next series of figures (Figs. 2-11) clearly shows this. Indeed, deep-space satellites can easily be isolated in orbital element space; see Table 1. One small, but rapidly growing, entry is debris caused by ARIANE related launch failures. As this has not received much treatment, I turn to a discussion of it.

\section{TABLE I HEAVILY POPULATED DEEP-SPACE ORBITS}

\begin{tabular}{lcccccc}
\hline Type & Mean Motion & Eccen. & Incl. & No. & Frac. \\
& & & & & & \\
\hline Nearly-Stationary & $0.9-1.1$ & $<0.05$ & $<10$ & 550 & 0.234 \\
Ariane & $2-6$ & $0.60-0.75$ & $<15$ & 400 & 0.170 \\
Cape Canaveral Rocket & Body $2-6$ & $0.60-0.75$ & $20-35$ & 300 & 0.128 \\
Molniya-Cosmos* & $1.75-2.25$ & $0.60-0.75$ & $60-75$ & 500 & 0.213 \\
Other A & $1.5-2.5$ & $0.60-0.75$ & $40-60$ & 150 & 0.064 \\
Other B & $2-6$ & $0.4-0.6$ & $10-20$ & 150 & 0.064 \\
Other C & $2-6$ & $0.4-0.6$ & $35-60$ & 150 & 0.064 \\
Other D & $2-6$ & $0.4-0.6$ & $90-120$ & $\underline{150}$ & 0.064 \\
& & & & & 2350 &
\end{tabular}

* Also strongly grouped in argument or perigee, $265^{\circ}-320^{\circ}$.

\section{ARIANE-RELATED DEBRIS}

I have searched a combination of a data bases for deep-space artificial satellites with orbital element sets with the following properties: (1) A mean motion within $1.5 \mathrm{rev} /$ day of 2.2 revolution per day; (2) an eccentricity within 0.3 of 0.73 ; and (3) an inclination within $3^{\circ}$ of 6.2 degrees. These are the critical orbital elements of a 1986 ESA rocket body. The inclination, longitude of the ascending node, and Gabbard diagrams for these objects are shown in Figs. 12, 13 , and 14.

The inclination distribution shows a broad, possibly multimodal form, centered near the ARIANE launch latitude. The unshown wings go further southward than they do northward. The longitude of the ascending node distribution shows a simple bimodal form with peaks on either side of the ARIANE launch longitude.

A Gabbard diagram is a plot of apogee and perigee heights against period. Thus, each satellite appears twice. When one artificial satellite breaks up into 
many pieces, the Gabbard diagram of its associated debris has an oblique $\mathrm{X}$ structure. The arms cross at the original orbital period and height. This structure is present in Fig. 14 and is a consequence of conservation of energy.

The eccentricity (Fig. 15) and argument of perigee (Fig. 16) distributions are less interesting. The nearly uniform aspect of the latter distribution is puzzling.

In summary, the result of a variety of optical searches for deep-space artificial satellites, when coupled with routine radar detection of deep-space satellites with low perigee heights, points to the rapid growth of objects in the ARIANE-type orbit $\left(\mathrm{n} \simeq 2 \mathrm{rev} / \mathrm{day}, \mathrm{e} \simeq 0.7, \mathrm{i} \simeq 7^{\circ}\right)$. These objects have appeared in a time frame consistent with explosions of ARIANE launch vehicles. Approximately 250 objects are now being tracked (as of January 1988).

\section{SUMMARY}

Searches for and detections of artificial satellite debris at a variety of wavelengths leads to two general conclusions. The first is that debris is concentrated in the same parts of orbital element space already occupied by rocket bodies and payloads. The second is that it is present in large numbers compared to the cataloged population. Tracking it is impossible for the current Spacetrack System.

\section{REFERENCES}

J. K. Beatty, Sky and Telescope 63, 469, 1982.

L. G. Taff, "Optical Artificial Satellite Searches," Project Report ETS-44, Lincoln Laboratory, MIT, 1979.

L. G. Taff, Publ. Astr. Soc. Pac 93, 618, 1981.

L. G. Taff, "Optical Search Theory and Optical Artificial Satellite Searches," Project Report ETS-64, Lincoln Laboratory, MIT., 1982.

T. G. Taff, Icarus 57, 259, 1984.

L. G. Taff, "The Analysis of Near-Earth Satellite Astrometric Data at the ETS," Project Report ETS-76, Lincoln Laboratory, MIT, 1985.L. G. Taff, J. Spacecraft and Rockets 23, 342, 1986.

L. G. Taff, "A GEODSS Search Exercise: Search Plan," Project Report STK-145 I, Lincoln Laboratory, MIT 1987a.

L. G. Taff, "A GEODSS Search Exercise: Results,"Project Report STK-145 II, Lincoln Laboratory, MIT 1987b.

L. G. Taff, "Ariane-Related Debris in Deep-Space Orbit," Project Report STK146, Lincoln Laboratory, MIT, 1987c.

L. G. Taff, D. E. Beatty, A. J. Yakutis, and P.M.S. Randall, Adv. Space Res. 6, $131,1986$.

L. G. Taff and D. M. Jonuskis, "Zoom us Full-Field Sensitivity at the ETS," Project Report ETS-78, Lincoln Laboratory, MIT, 1987.

R. Weber, SPIE 203, 6, 1979a.

R. Weber, Opt. Eng. 18, 82, 1979 b.

A. J. Yakutis, L. G. Taff, and S. Sayer, "Calibration of ETS Videotapes," Project Report ETS-77, Lincoln Laboratory, MIT, 1986. 


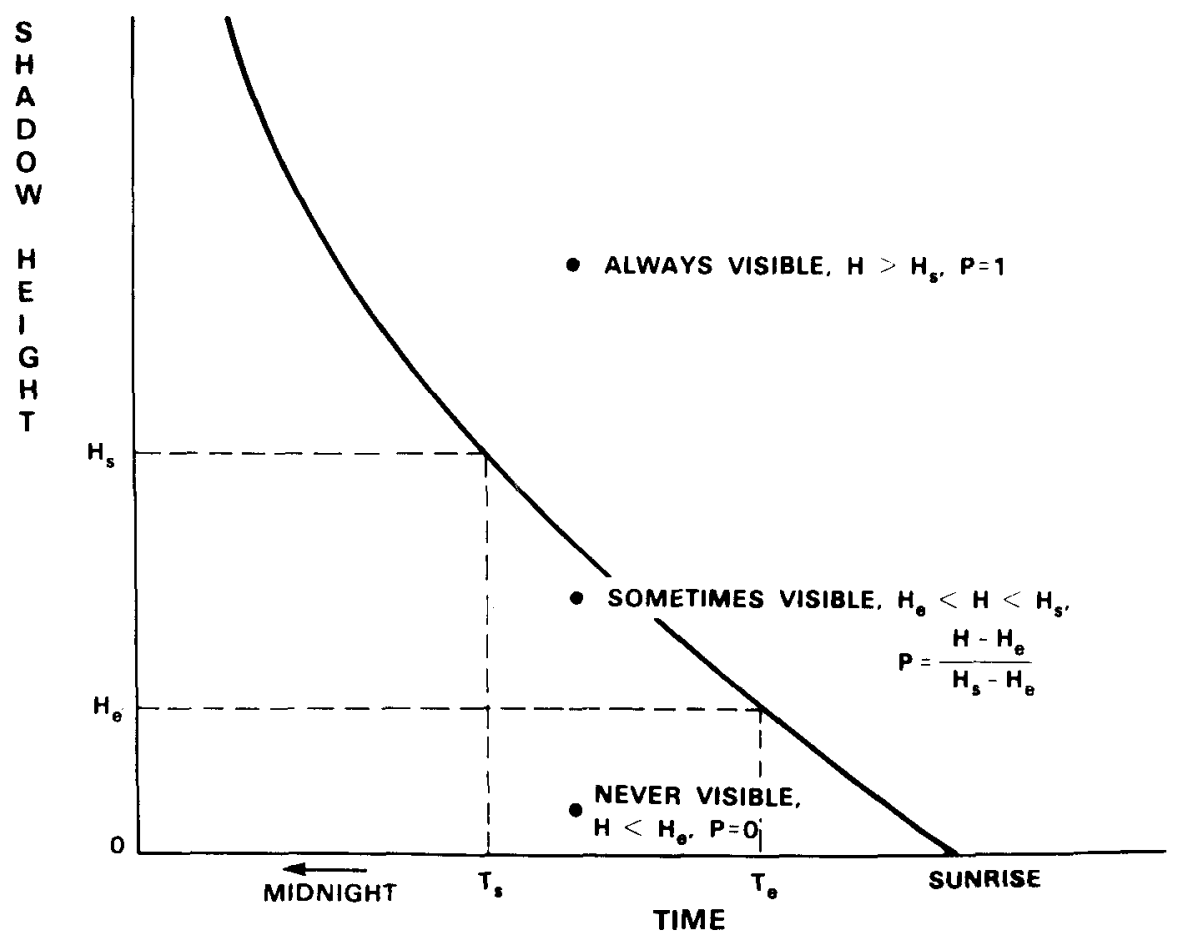

1. Representative shadow height vs time curve (post-midnight to dawn). 


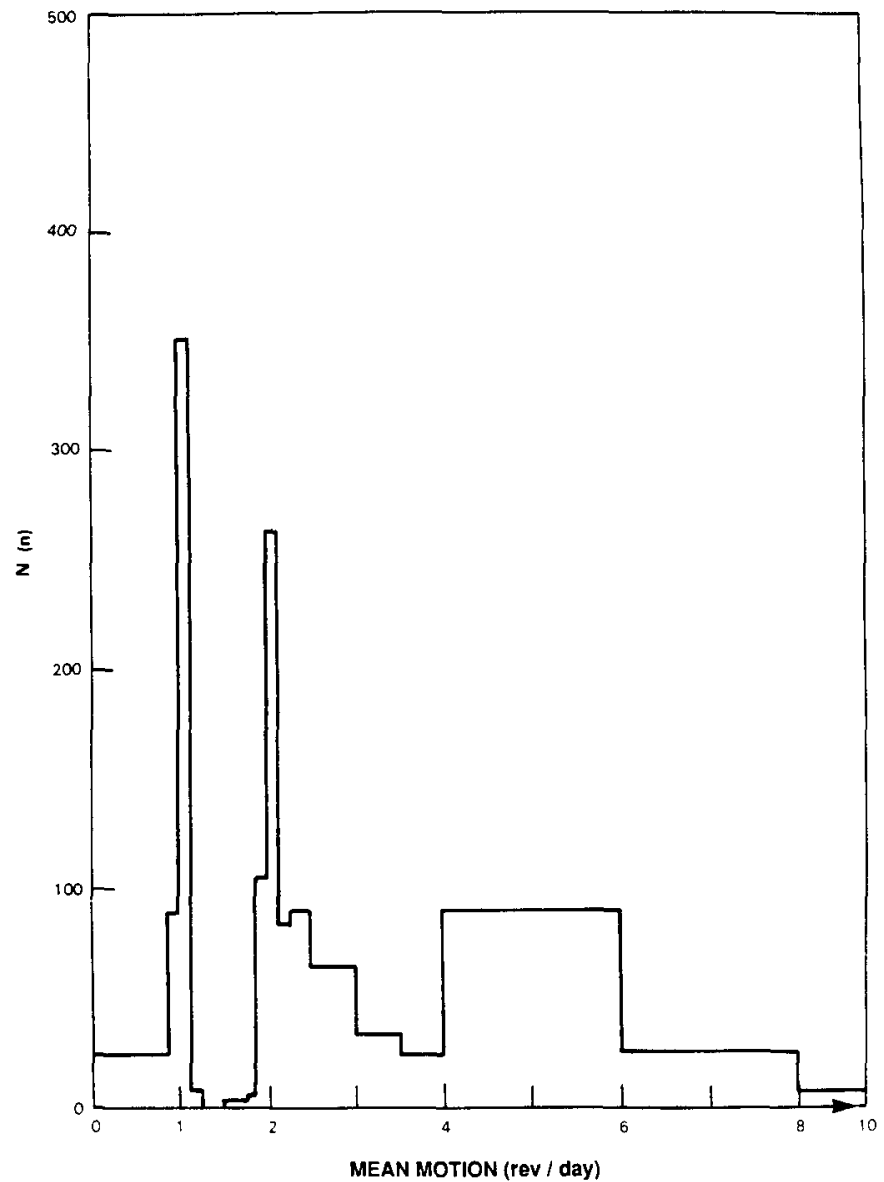

2 Mean motion distribution for all cataloged deep-space artificial satellites. Note the near-stationary peak near $n=1$, the Cape Canaveral Rocket Body/Molniya-Cosmos peak near $n=2$, and the lower rocket bodies and junk beyond $n=4$. 


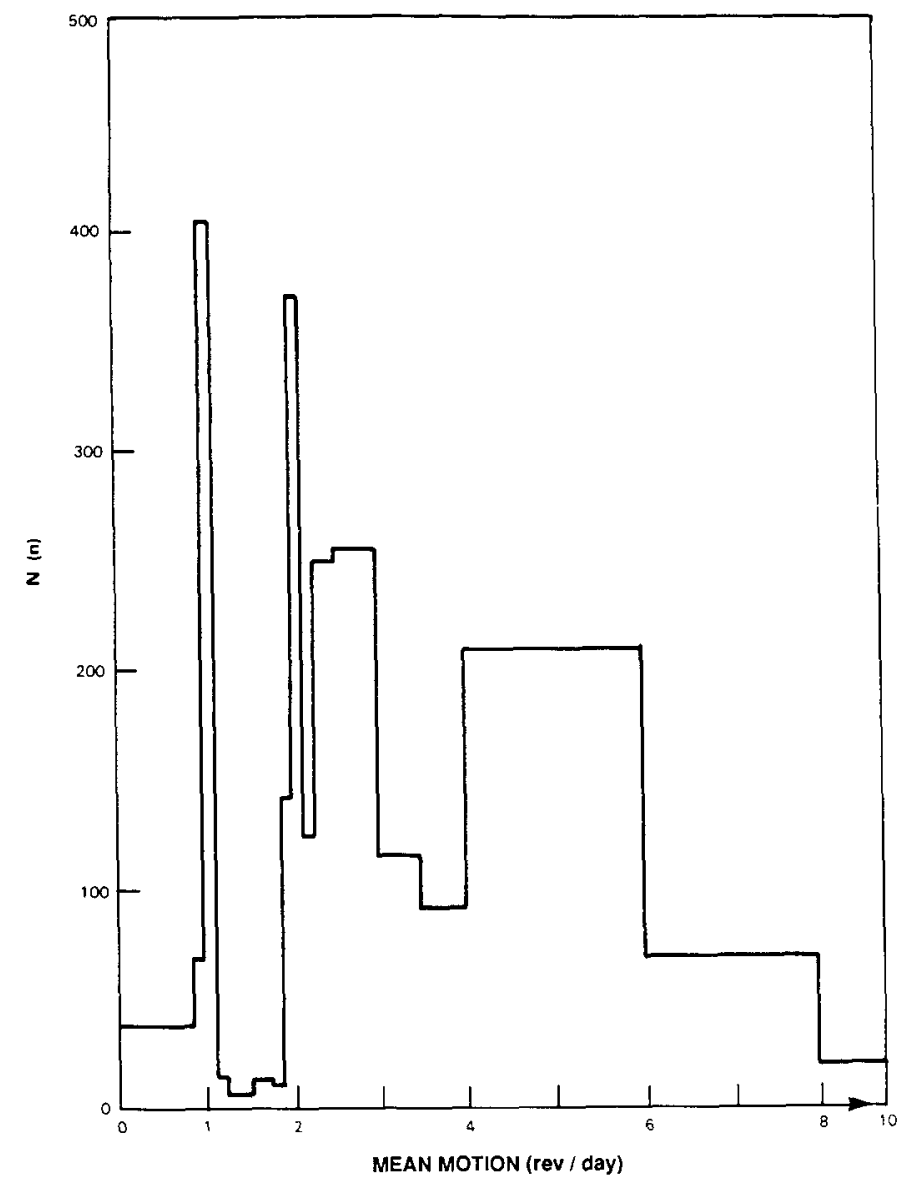

3. Same as Fig. 2 but for uncataloged (but tracked) deep-space satellites. Note the same peaks but the broader widths. This is partly a consequence of operational factors and less accurate orbital elements sets. 


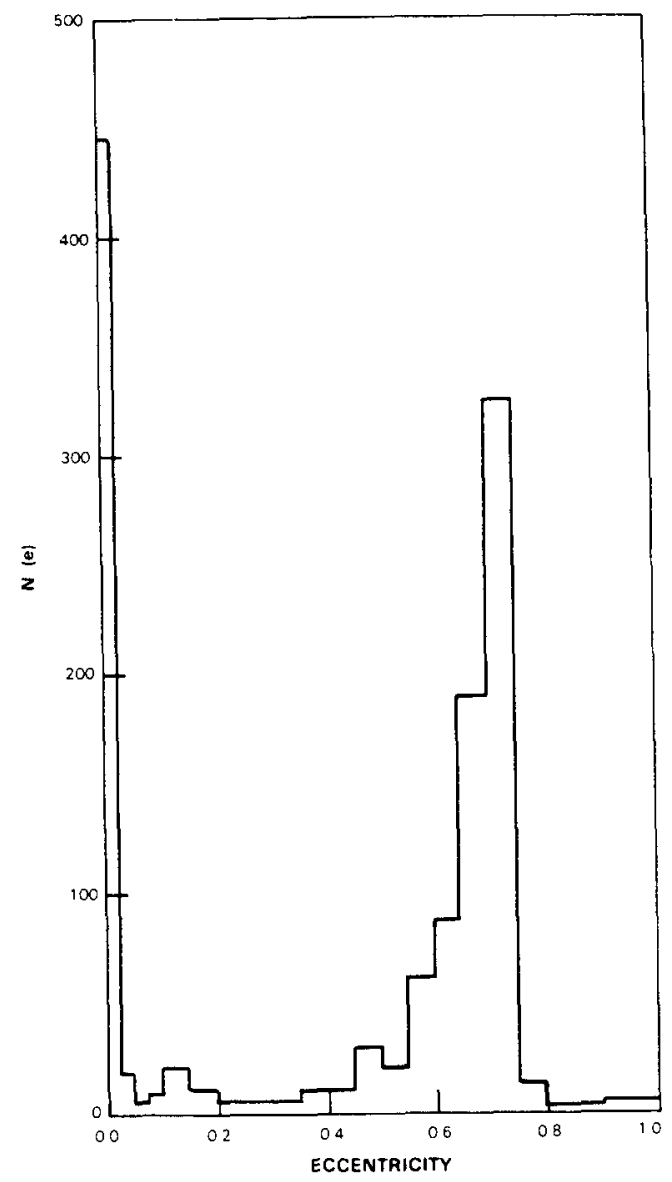

4. Same as Fig. 2 but for eccentricity. Note the nearly circular peak (which represent satellites that are mostly nearly-stationary too) and the Molniya-Cosmos peak. 


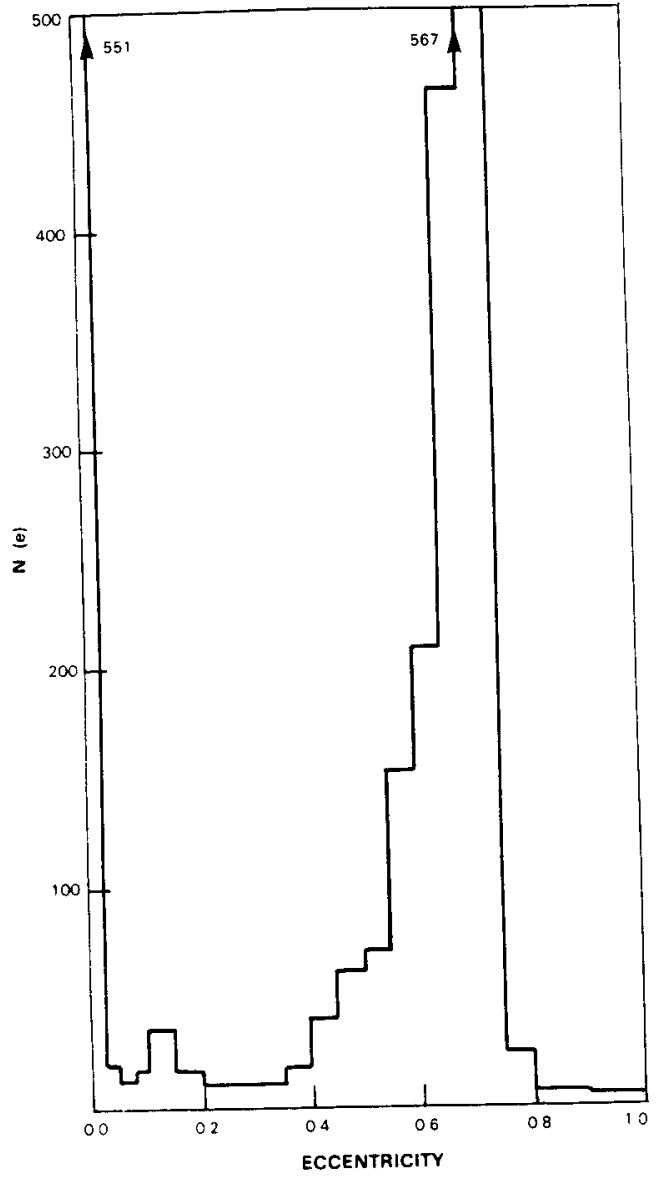

5. Same as Fig. 3 but for eccentricity. 


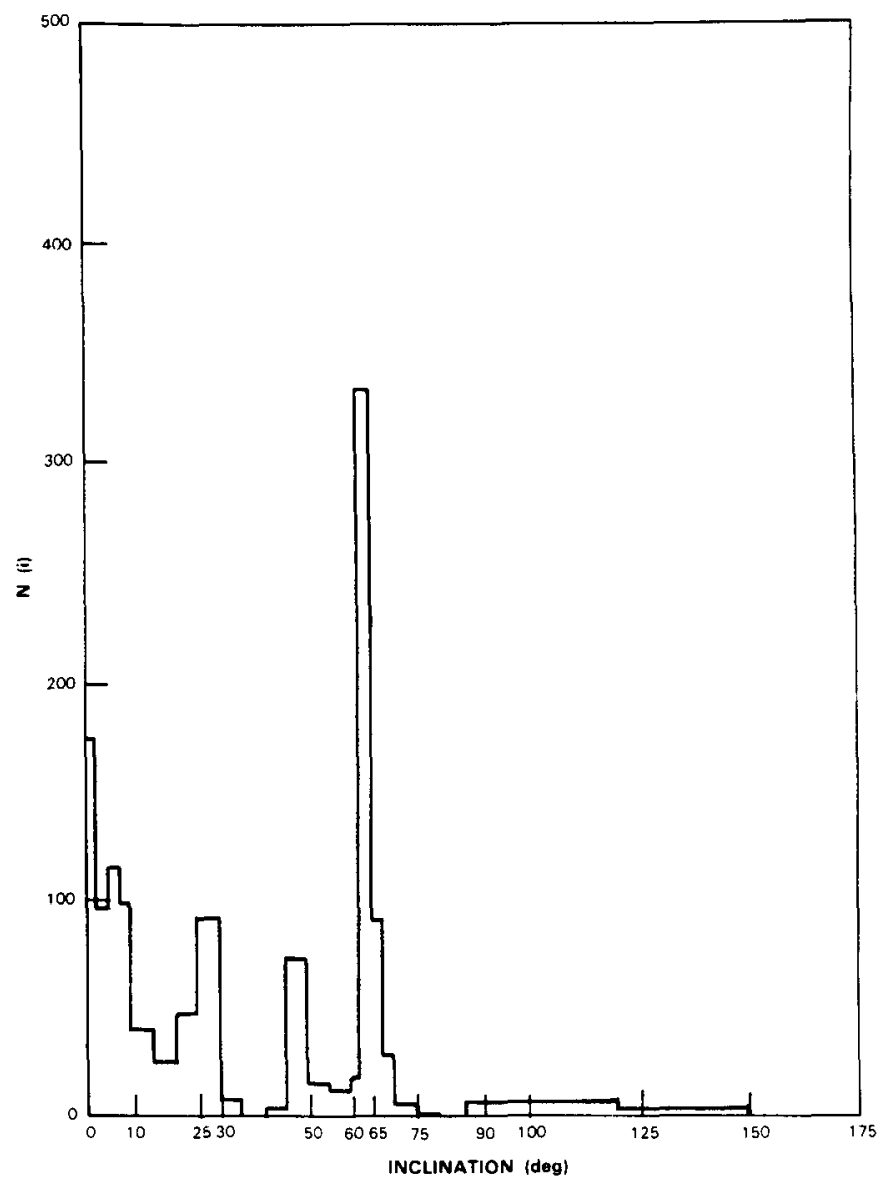

6. Same as Fig. 2 but for inclinations. Once again the Molniya-Cosmos group stands out as do the Cape Canaveral Rocket bodies and the nearly-stationary satellites. 


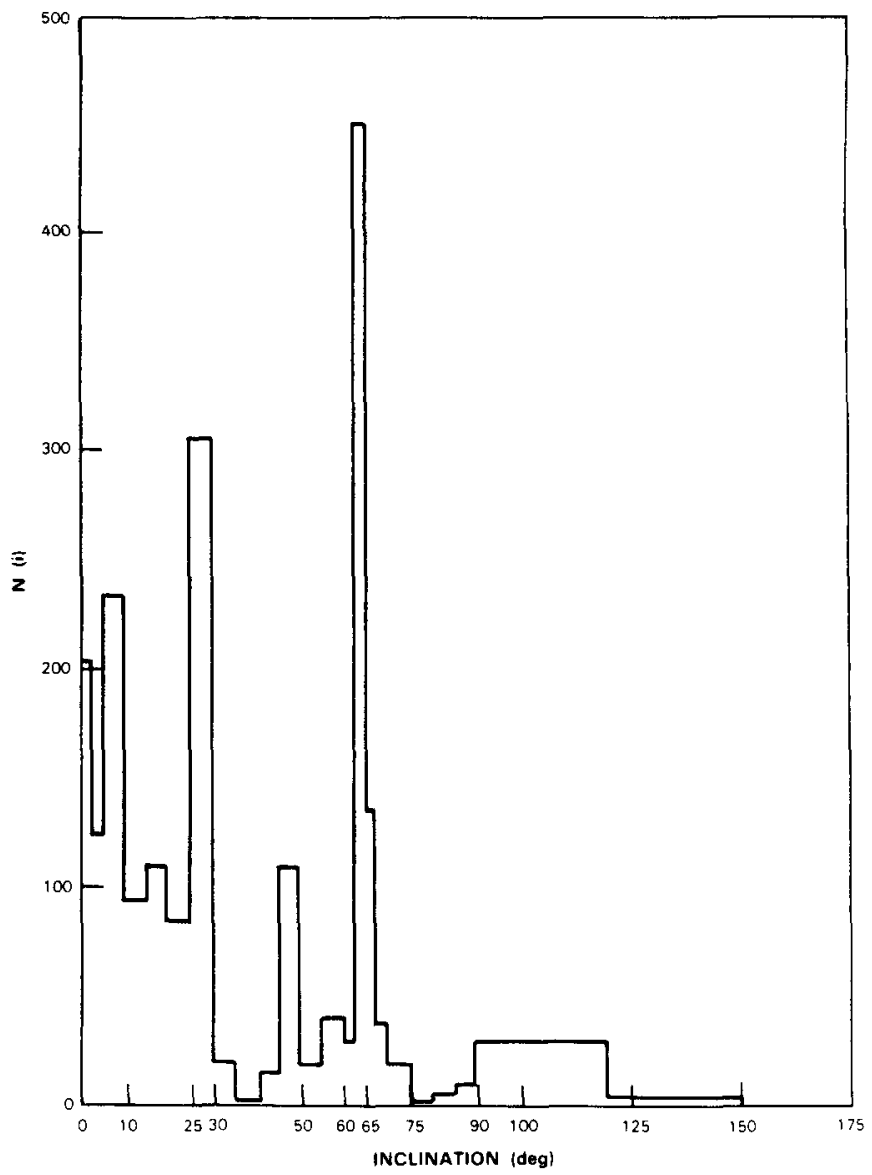

7. Same as Fig. 3 but for inclination. 